\title{
Efecto del EDTA sobre la toxicidad del cromo (VI) en el desarrollo larval de Petrolisthes laevigatus (Guérin, 1835)
}

\author{
Effect of EDTA on chromium (VI) toxicity on larval development of \\ Petrolisthes laevigatus (Guérin, 1835) \\ María Paz Lermanda1, Carolina Urrutia' ${ }^{1}$, Vanessa Novoa ${ }^{1}$ y Anny Rudolph ${ }^{1}$ \\ ${ }^{1}$ Departamento de Química Ambiental, Facultad de Ciencias, Universidad Católica de la Santísima Concepción, \\ Casilla 297 Concepción, Chile \\ annyr@ucsc.cl
}

\begin{abstract}
The larval development and growth of Petrolisthes laevigatus were analyzed in five concentrations of Cr VI, 0 (control), 0.25, 0.5, 1, and $5 \mathrm{mg} \mathrm{L}^{-1}$ chrome (VI) in filtered sea water and the same chrome (VI) concentrations, plus $0.7 \mathrm{mg} \mathrm{L}^{-1}$ EDTA. Analyses were done during 21-day period. Measurement of total length, individual wet average larvae weight and the presence or absence of deformations in $P$. laevigatus larvae were determined. The larvae of the control group ( $0 \mathrm{mg} \mathrm{L}^{-1}$ chrome (VI)) did not exhibit deformations and developed normally. The total length and biomass of the larvae exposed to Cr VI were significantly lower than those of the larvae subjected to Cr VI plus EDTA concentrations and the control larvae. It is hereby confirmed that the presence of EDTA in the medium mitigates the negative effects of $\mathrm{Cr} \mathrm{VI}$ via a yet unknown mechanism.
\end{abstract}

Key words: Crab, zoea larva, toxicity, Cr (VI) + EDTA
Resumen.- Se analizó el desarrollo larval y el crecimiento de Petrolisthes laevigatus en cinco concentraciones de $\mathrm{Cr}$ (VI): 0 (control), 0,$25 ; 0,5 ; 1$ y $5 \mathrm{mg} \mathrm{L}^{-1}$ de cromo (VI) en agua de mar filtrada y las mismas cinco concentraciones de cromo (VI) más la adición de $0,7 \mathrm{mg} \mathrm{L}^{-1}$ de EDTA. El estudio se condujo en 21 días. Se determinó longitud total, peso promedio y presencia o ausencia de malformaciones en larvas de $P$. laevigatus. Las larvas de las unidades control ( $0 \mathrm{mg} \mathrm{L}^{-1}$ de cromo (VI)) no presentaron malformaciones y se desarrollaron en forma normal. La longitud total y peso promedio de las larvas expuestas a $\mathrm{Cr}$ (VI) fueron significativamente menores con respecto a los de las larvas sometidas a las concentraciones de Cr (VI) más EDTA y a las unidades control. Se confirma que la presencia de EDTA en el medio atenúa los efectos negativos del Cr (VI) por un mecanismo aún no dilucidado.

Palabras clave: Cangrejo, larva zoea, toxicidad, Cr (VI) + EDTA

\section{Introducción}

Los metales vertidos en fuentes de agua han tenido efectos negativos, en distintos grados, en el crecimiento, supervivencia y desarrollo de las especies que allí habitan. Principalmente son las primeras etapas del ciclo de vida de los organismos las que sufren los efectos negativos, en el tamaño de la primera reproducción, número de episodios reproductivos, ontogenia en las fases posteriores y longevidad de los organismos (Hadfield \& Strathmann 1996, Natale et al. 2000, Boada et al. 2007). Es así que resulta preocupante el aumento de residuos con metales eliminados en la zona costera y el efecto que puede causar en las especies y el medioambiente.

El cromo es un metal que ingresa como integrante de residuos líquidos al medio acuático, debido a su utilización en actividades tan diversas como: la industria textil, cueros, industrias de papel y cartón, petroquímica, abonos, refinerías, metalúrgica, vidrios, cementos, asbestos, pinturas, cerámicas, fungicidas, catálisis y galvanizados (Bodar et al. 1990, Rengaraj et al. 2001).
Por ejemplo, un área que representa un segmento importante de la economía chilena, como Bahía San Vicente, recibiría a través de los efluentes industriales una carga de cromo estimada en $3,12 \mathrm{Kg} / 20 \mathrm{~h}$, generando en los sedimentos del área concentraciones de 128,81 \pm $1,10 \mu \mathrm{g} \mathrm{g}^{-1}$ (Ahumada \& Vargas 2005); concentraciones mayores se han observado en sectores de los sedimentos de la cuenca del Río Salado en Argentina, i.e. $260 \mu \mathrm{g} \mathrm{g}^{-1}$ (Gagneten et al. 2007).

Las concentraciones de cromo en el ambiente marino superiores al nivel basal generan respuestas sensibles y específicas en los crustáceos, como malformaciones i.e. desarrollo incompleto de maxilipedos, atrofia del telson, espina dorsal y/o espina caudal (Amin 1995). Además, en el cangrejo Barytelphusa guerin se observó que, tanto a su forma trivalente como hexavalente, el cromo generó una drástica reducción de los niveles de glucógeno, con resultados nefrotóxicos (Sridevi \& Reddy 2000).

Los crustáceos decápodos, al igual que otros organismos, han desarrollado estrategias de supervivencia 
al estrés generado por los contaminantes, lo que les ha permitido sobrevivir en una variedad de hábitats (Pinheiro \& Fransozo 1995). Aspectos de la biología reproductiva como la producción de huevos, desarrollo larval y la estimación de fecundidad se estudian con el objeto de comprender y asegurar la renovación natural de sus poblaciones, como también para efectuar un manejo adecuado en aquellas especies de importancia comercial (Caddy 1989, Scelzo 1997, Hernández \& Palma 2003, González-Pizani et al. 2006).

Se ha demostrado que las larvas pueden presentar plasticidad fenotípica como respuesta a alteraciones del ambiente (Hadfield \& Strathmann 1996). Vonesh \& Miner (2004) demostraron que las larvas no sólo pueden alterar su morfología en respuesta a diferencias en la concentración de nutrientes, sino también a variaciones en la concentración de metales en el medio acuático. La respuesta más común de los crustáceos expuestos a contaminantes metálicos es la inhibición de la ecdisis y un aumento en el periodo de desarrollo larval (Lombardo et al. 1982, Fingerman 1985). Para larvas planctotróficas, la calidad ambiental del medio en el cual se desarrollan tiene directa relación con la supervivencia, crecimiento y desarrollo larval, condicionando el reclutamiento y la dispersión de la especie (Levin 2006). Existen estudios en los que se ha detectado efectos deletéreos cuantificables sobre el crecimiento y supervivencia de crustáceos por la acción de cantidades residuales de plaguicidas y de iones metálicos (Petriella \& Boschi 1997, Urrutia et al. 2008).

Se postula que en el medio acuático los efectos nocivos de los metales sobre los organismos pueden ser modulados por la presencia de agentes quelantes naturales y artificiales (Sperling \& Welz 1992). Los quelantes disminuirían el efecto deletéreo de los iones, siendo capaces de reducir la producción acelerada y excesiva de radicales libres que producen degeneración celular (Espinoza et al. 2003). Entre los quelantes más estudiados a nivel de laboratorio se encuentra el ácido etylendiaminotetracetico (EDTA). Su utilización durante las etapas de cría y cultivo de organismos acuáticos facilitaría procesos de muda en crustáceos, reduciendo malformaciones y/o mortalidad (Scelzo 1998).

Se estudió la factibilidad de utilizar como organismo blanco en ensayos de toxicidad, el desarrollo larval y crecimiento de las larvas en estado de zoea del cangrejo Petrolisthes laevigatus (Guérin, 1835), especie que se distribuye en las costas del Pacífico sudeste desde Perú al Canal Messier en Aysén, Chile (Urrutia et al. 2008). Este cangrejo presenta un desarrollo larval planctónico, que consiste luego de la eclosión, en un estado de prezoea, dos zoeas y una megalopa (Albornoz \& Wehrtmann 1996, Lardies \& Wehrtmann 1996). En larvas zoea de esta especie, se analizó su respuesta biológica en presencia de concentraciones de $\mathrm{Cr}$ (VI), concentraciones de $\mathrm{Cr}$ (VI) en que se ha adicionado EDTA bajo el supuesto que las larvas en estado de zoea podrían ser recolectadas sin sufrir daño y que la acción tóxica del Cr (VI) sería disminuida ante la presencia de quelantes en el medio.

\section{Material y métodos}

Se recolectaron alrededor de 500 hembras ovígeras de $P$. laevigatus, durante baja marea en el intermareal de un sector de la playa de bolones en Lenga (36 $45^{\circ} \mathrm{S}$ $70^{\circ} 10^{\prime} \mathrm{W}$ ), con baja influencia humana (Moscoso et al. 2006, Altamirano-Chovar et al. 2006), en octubre de 2006. Se seleccionaron hembras que presentaban huevos en estadio III avanzado (Lardies \& Wehrtman 1996), las cuales fueron ubicadas en cubetas con agua de mar filtrada (1 $\mu$ ) y alimentadas cada $48 \mathrm{~h}$ con nauplios de Artemia salina obtenidos a partir de huevos recién eclosionados en el laboratorio (González-Pizani et al. 2006).

Las hembras fueron ubicadas en un sistema diseñado especialmente para una recolección cuidadosa y sin destrucción de las larvas, que permitía la recirculación suave del agua, haciéndola pasar a través de un tamiz, en el cual las larvas eran recolectadas. Pasado 4 días se seleccionaron las larvas de P. laevigatus en estado zoea I con notoria movilidad y buen estado. Las larvas fueron mantenidas en laboratorio para su aclimatación por 7 días, a $17 \pm 1^{\circ} \mathrm{C}, \mathrm{pH} 8,2 \pm 0,1$, fotoperiodo de $12: 12 \mathrm{~h}$, salinidad entre 33-34 ups, aireación constante y alimentadas cada dos días con nauplios de Artemia recién eclosionados.

Se dispusieron 30 unidades de investigación (= bandejas) de $500 \mathrm{~mL}$ de capacidad, cada una con $200 \mathrm{~mL}$ de agua de mar filtrada $(1 \mu)$ y 90 larvas. Quince bandejas fueron separadas en 5 grupos (3 réplicas en cada una) para realizar el tratamiento con $\mathrm{Cr}(\mathrm{VI})$ : 0 (control), 0,25, $0,5,1$ y $5 \mathrm{mg} \mathrm{L}^{-1}$, las cuales fueron preparadas por dilución desde una solución madre de $1000 \mathrm{mg} \mathrm{L}^{-1}$ de $\mathrm{Cr}$ (VI) en agua de mar filtrada ( $\mathrm{Cr}(\mathrm{VI}): \mathrm{K}_{2} \mathrm{Cr}_{2} \mathrm{O}_{7}$, Índice Merck 104.862). Las otras quince bandejas correspondieron al tratamiento realizado con las mismas concentraciones de cromo (VI) i.e. 0 (control), 0,25 $\mathrm{mg} \mathrm{L}^{-1}, 0,5 \mathrm{mg} \mathrm{L}^{-1}, 1 \mathrm{mg}$ $\mathrm{L}^{-1}$ y $5 \mathrm{mg} \mathrm{L}^{-1}$; más la adición en cada unidad de EDTA, en una concentración nominal de $0,7 \mathrm{mg} \mathrm{L}^{-1}$ (sal sódica del ácido etilenaminotetracético: Índice Merck 324.503), concentración inferior a la utilizada por Scelzo (1998), i.e. $1 \mathrm{mg} \mathrm{L}^{-1}$, en que comparaban desoves y supervivencia larval del camarón Artemisia longinaris en agua de mar con EDTA y en agua de mar sin EDTA. Se utilizó, de acuerdo al diseño experimental, un total de 2700 larvas. 
El ensayo se condujo durante 21 días, de acuerdo al trabajo de Lardies \& Wehrtman (1996), observando diaria y cuidadosamente el desarrollo de los controles.

Las variables de respuesta medidas en las larvas de estado de zoea I fueron tres: longitud corporal total, peso estimado promedio y malformaciones. Al inicio y al término del experimento se registró la longitud total mediante un retículo graduado bajo microscopio óptico, el peso promedio fue medido en peso húmedo de las larvas, determinado con una balanza analítica de 0,0001 g de precisión, y se observó la morfología de las larvas (en cada unidad experimental) bajo microscopio óptico, para determinar la presencia de malformaciones i.e. en el telson y atrofia de la espina dorsal. Se contó el número de organismos con malformaciones y su valor fue expresado en porcentaje para cada tratamiento.

\section{Análisis estadísticos}

Se utilizó el programa STATISTICA versión 6.0 (2001). Se aplicó la prueba de Shapiro Wilks para probar normalidad de los datos y la prueba de Cochran para observar homogeneidad de la varianza. Se aplicó una prueba ANDEVA de dos vías factorial (tratamiento y concentraciones) con el objetivo de analizar si existían diferencias entre las variables de respuesta en las distintas concentraciones de los dos tratamientos. Finalmente, se aplicó una prueba a posteriori de Tuckey para identificar las concentraciones en que el cromo generaría diferencias significativas respecto del control.

\section{Resultados}

\section{Crecimiento larval}

Las larvas de las unidades control ( $0 \mathrm{mg} \mathrm{L}^{-1}$ de $\left.\mathrm{Cr} \mathrm{VI}\right)$, con agua de mar filtrada y con agua de mar filtrada más EDTA, presentaron una longitud total promedio de 7,59 $\pm 0,13$ y 7,62 $\pm 0,07 \mathrm{~mm}$, respectivamente. La longitud total de las larvas sometidas a distintas concentraciones de $\mathrm{Cr}$ (IV) (i.e., 0,25, 0,5, 1 y $5 \mathrm{mg} \mathrm{L}^{-1}$ ), fue significativamente menor con respecto a la longitud total de las larvas sometidas a las concentraciones de $\mathrm{Cr}(\mathrm{VI})$ más EDTA (ANOVA 2 vías; $\mathrm{F}=13,28$; g.l. $=4 ; P<0,001$ ) (Fig. 1).

\section{Peso promedio larval}

Las larvas de las unidades control $\left(0 \mathrm{mg} \mathrm{L}^{-1} \mathrm{de} \mathrm{Cr} \mathrm{VI}\right)$, presentaron un peso promedio estimado en $0,0079 \pm$ 0,0001 y de $0,0081 \pm 0,0003 \mathrm{~g}$ ind.$^{-1}$, respectivamente. En la Fig. 2 se observa que el peso promedio de las larvas a partir de la concentración $0,25 \mathrm{mg} \mathrm{L}^{-1}$ en el tratamiento con $\mathrm{Cr}(\mathrm{VI})$, disminuyó significativamente con respecto

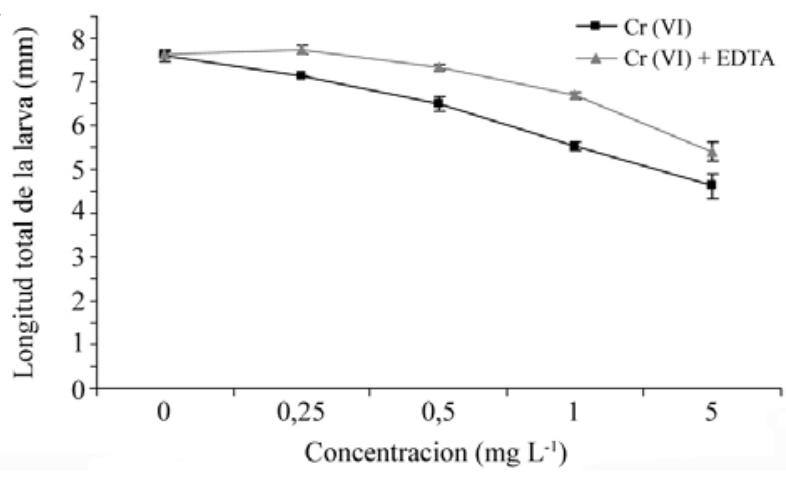

Figura 1

Longitud total promedio ( \pm d.s.) de larvas de P. laevigatus control y en las distintas concentraciones de Cr VI y Cr VI más EDTA $(\mathbf{n}=90)$

Average of total length ( \pm s.d.) of $P$. laevigatus for control larvae and larvae kept in different concentrations of $\mathrm{Cr}$ VI and Cr VI plus EDTA $(\mathrm{n}=90)$

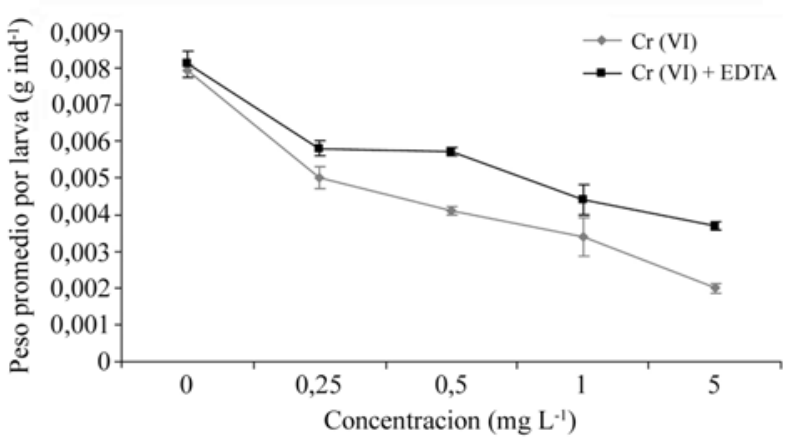

Figura 2

\section{Biomasa promedio ( \pm d.s.) de las larvas de P. laevigatus control y) y en las distintas concentraciones de Cr VI y Cr VI más EDTA $(\mathbf{n}=90)$}

Average of biomass ( \pm s.d.) for larvae of P. laevigatus kept in different concentrations of $\mathrm{Cr}$ VI and Cr VI plus EDTA $(n=90)$

a las larvas del grupo control, con un valor de $0,0050 \pm$ $0,0003 \mathrm{~g}_{\text {ind. }}{ }^{-1}$ alcanzando en la concentración de $5 \mathrm{mg}$ $\mathrm{L}^{-1} \mathrm{Cr}$ (VI) un peso promedio de $0,0020 \pm 0,0001 \mathrm{~g}$ ind.$^{-1}$, siendo su peso promedio significativamente menor respecto al peso promedio de las larvas sometidas a todas las concentraciones de $\mathrm{Cr}$ (VI) más EDTA (Fig. 2) (ANOVA 2 vías; $\mathrm{F}=7,008 ; \mathrm{g} . \mathrm{l}=4 ; \mathrm{P}=0,001$ ). 


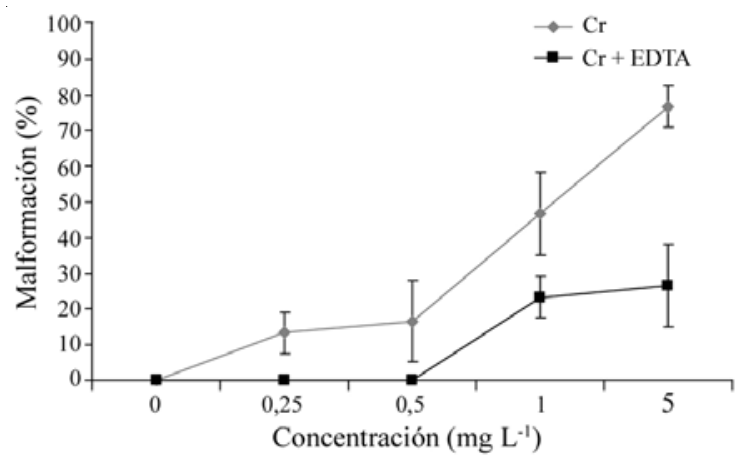

Figura 3

Porcentaje (\%) promedio ( \pm d.s.) de malformaciones de larvas de $P$. laevigatus sometidas a concentraciones crecientes de cromo (VI) y cromo (VI) más EDTA (n=90)

Mean percentage $(\%)( \pm$ d.s.) of malformations on larvae of $P$. laevigatus under increasing concentrations of chromium (VI) and chromium (VI) plus EDTA $(\mathrm{n}=90)$

\section{Desarrollo larval}

Las larvas en ambos grupos controles $\left(0 \mathrm{mg} \mathrm{L}^{-1} \mathrm{de} \mathrm{Cr}\right.$ VI), no presentaron malformaciones (Figs. 4 y 5). Se observaron malformaciones en las larvas sometidas a partir de concentraciones de $0,25 \mathrm{mg} \mathrm{L}^{-1}$ de cromo (VI) con un porcentaje del $13,3 \pm 5,77 \%$ y en los tratamientos con cromo (VI) más EDTA a partir de la concentración de $1 \mathrm{mg} \mathrm{L}^{-1}$ con un promedio del 23,3 \pm 5,73\% (Fig. 3). Las larvas sometidas a las concentraciones crecientes de cromo (VI) presentaron un aumento significativo de malformaciones en relación a las sometidas a las concentraciones de cromo (VI) más EDTA y controles (ANOVA 2 vías; $\mathrm{F}=10,23 ;$ g.1. $=4 ; P=0,001$ ). Las malformaciones más comunes observadas fueron: atrofia del telson (Fig. 4), atrofia de la espina dorsal (Fig. 5) y malformación completa de las larvas.

\section{Control}
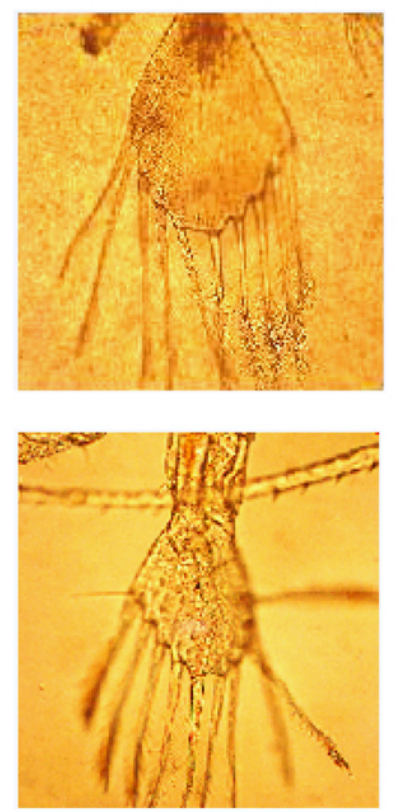

\section{Cromo (VI) + EDTA}
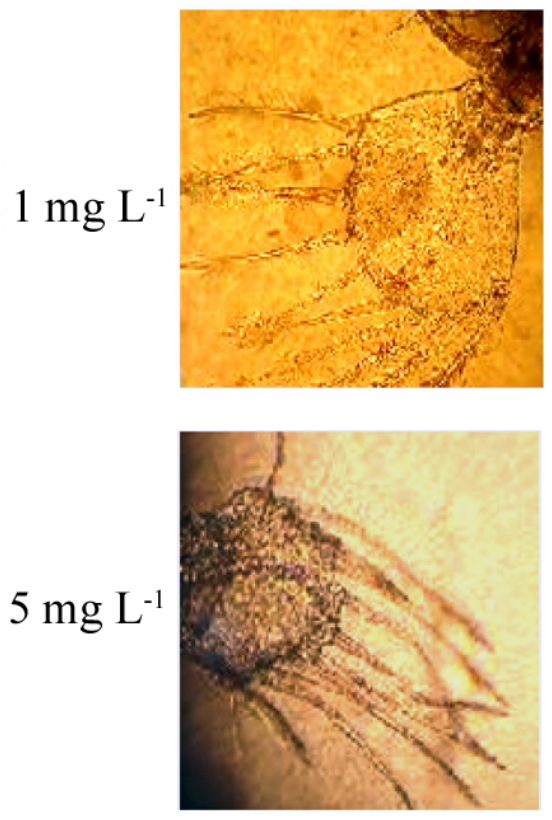

\section{Cromo (VI)}
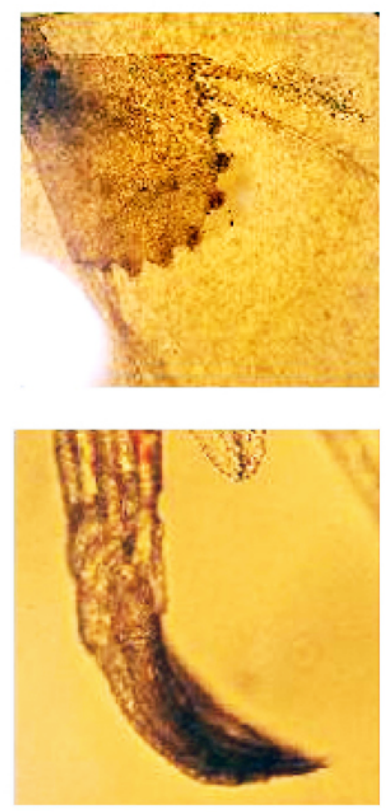

Figura 4

Telson normal en larvas en el medio control de $P$. laevigatus y telson anormal de larvas sometidas a concentraciones de 1 y $5 \mathrm{mg} \mathrm{L}^{-1}$ de Cr VI más EDTA y 1 y $5 \mathrm{mg} \mathrm{L}^{-1} \mathrm{Cr}$ VI

Normal telsons of $P$. laevigatus larvae (control) and affected telson of larvae kept at 1 and $5 \mathrm{mg} \mathrm{L}^{-1}$ of Cr VI plus EDTA, and 1 and $5 \mathrm{mg} \mathrm{L}^{-1}$ of Cr VI 


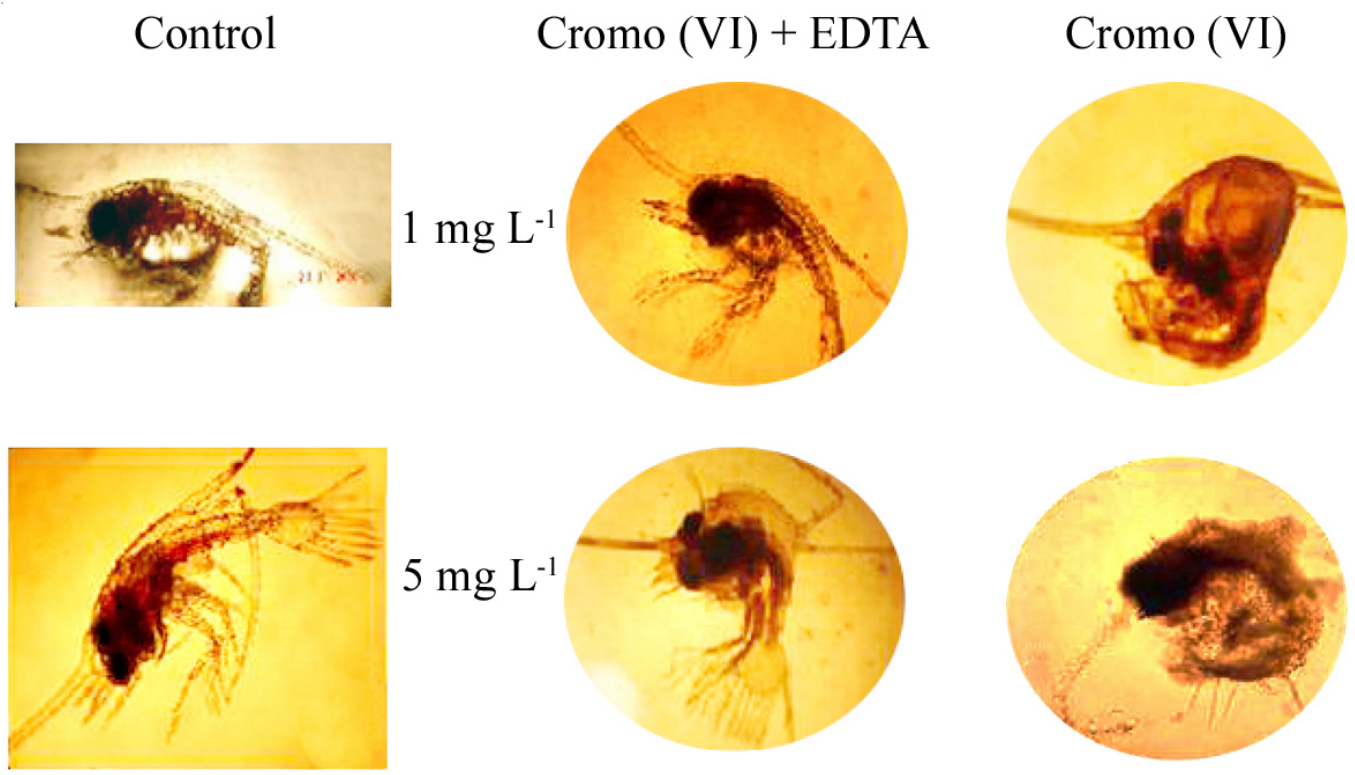

Figura 5

Larvas control de $P$. laevigatus y atrofia de la espina dorsal observada en las larvas sometidas a concentraciones de 1 y $5 \mathrm{mg} \mathrm{L}^{-1}$ de Cr VI más EDTA y de 1 y $5 \mathrm{mg} \mathrm{L}^{-1} \mathrm{Cr}$ VI

Control $P$. laevigatus larvae and atrophy of the dorsal spine observed in the larvae kept at 1 and $5 \mathrm{mg} \mathrm{L}^{-1}$ of Cr VI plus EDTA and 1 and $5 \mathrm{mg} \mathrm{L}^{-1}$ of Cr VI

\section{Discusión}

Los estadios tempranos del desarrollo de los organismos son períodos de rápido crecimiento y de división celular, representando un estado sensible de un ciclo de vida (Antezana et al. 1965), motivo por el cual se utilizan para analizar situaciones de estrés ambiental (Dorigan \& Harrison 1979), como por ejemplo, presencia de iones metálicos en el medio por sobre los niveles basales.

Las larvas de Petrolisthes laevigatus en los tratamientos controles $\left(0 \mathrm{mg} \mathrm{L}^{-1} \mathrm{de} \mathrm{Cr} \mathrm{VI}\right)$, i.e. agua de mar filtrada y agua de mar filtrada más EDTA, presentaron un desarrollo normal en su peso promedio y longitud total, lo que indica que en un medio libre de agentes químicos, aún encontrándose en condiciones de laboratorio, se produce un desarrollo normal de las larvas, lo que está de acuerdo con lo observado en larvas de crustáceos por Amín (1995) y en adultos de crustáceos por Urrutia et al. (2008). Por otra parte, las larvas en estado de zoea de $P$. laevigatus han mostrado que son fáciles de recolectar y factibles de utilizar como organismo blanco en ensayos de toxicidad. En este estudio, las malformaciones observadas en las larvas de P. laevigatus, por efecto de la presencia en el medio de $\mathrm{Cr}$ (VI), coinciden con las malformaciones informadas en larvas del cangrejo Chasmagnathus granulata expuestas a concentraciones subletales de plomo i.e. atrofia de espina dorsal, de espinas laterales y telson (Keselman 2002).

En el medio acuoso el cromo puede encontrarse como $\mathrm{Cr}$ (III) y Cr (VI). El Cr (III), y en bajas concentraciones, es esencial para mantener procesos fundamentales de la vida como el metabolismo de la glucosa, lípidos y proteínas, tanto en humanos como en animales. Sin embargo, en altas concentraciones es tóxico (Warnick \& Bell 1969, Schneider \& Jefferson 1997, Natale et al. 2000). En cambio, al Cr (VI) no se le conocen acciones benéficas sobre los organismos y se define como tóxico, atribuyéndosele propiedades mutagénicas y cancerígenas (Threeprom et al. 2005, Ngah et al. 2006, Rai \& Mehrotra 2008).

La formación del complejo Cr (III)-EDTA se encuentra documentado en la literatura (Skoog et al. 2005). En cambio, el Cr (VI) sobre pH 7 (agua de mar), podría estar presente como iones cromato, dicromato, cromato ácido y/o ácido crómico (Sperling \& Welz 1992), formas aniónicas con muy baja probabilidad de reaccionar 
con EDTA en solución (Skoog et al. 2005, Threeprom et al. 2005). Sin embargo, en presencia de EDTA el crecimiento en longitud total de las larvas $P$. laevigatus fue mayor, en comparación a las larvas sometidas a concentraciones sólo de $\mathrm{Cr}$ (VI). Semejantes resultados se observaron también respecto del crecimiento en peso promedio de las larvas, con un 74\% de reducción en el peso promedio de las larvas expuestas a $5 \mathrm{mg} \mathrm{L}^{-1} \mathrm{de} \mathrm{Cr}$ VI comparado con el grupo control ( $0 \mathrm{mg} \mathrm{L}^{-1} \mathrm{de} \mathrm{Cr}$ VI) y un porcentaje menor, de sólo $54 \%$ con respecto a la misma concentración de $\mathrm{Cr}$ (VI) pero en presencia de EDTA.

Los resultados confirman que la presencia de EDTA atenúa los efectos negativos del $\mathrm{Cr}$ (VI) sobre las larvas en relación a su crecimiento (Fig. 1) y peso promedio (Fig. 2) y alteraciones morfológicas (Figs. 3, 4 y 5), lo que es coincidente con lo observado en ejemplares adultos de Petrolisthes laevigatus por Urrutia et al. (2008).

Scelzo (1998) plantea que el mecanismo de acción del EDTA, al disminuir la toxicidad del $\mathrm{Cr}$ (VI) en solución, es desconocido. La adición de EDTA en pequeñas concentraciones se utiliza como un mecanismo preventivo y efectivo en la larvicultura comercial de camarones, langostinos y cultivos de fitoplancton. $\mathrm{Su}$ adición permitiría controlar sustancias contaminantes de origen terrestre, doméstico y/o industrial que pueden estar presentes en el agua de mar, especialmente si se trabaja con agua de mar costera. Sin embargo, no se puede asumir que la adición de EDTA al agua de mar puede ser siempre beneficiosa, ya que su exceso disminuiría las cantidades de calcio por debajo de los óptimos necesarios para el cultivo (Lawrence et al. 1981) y en el caso de cultivos del alga Petrolisthes subcapitata, se atribuye a un exceso de EDTA la disminución en los contenidos necesarios de $\mathrm{Fe}^{+3}$ biodisponible (Lee et al. 2009).

Los resultados obtenidos permiten concluir que la adición de EDTA en concentraciones de $0,7 \mathrm{mg} \mathrm{L}^{-1}$ atenúa los efectos negativos del $\mathrm{Cr}$ (VI) presente en el medio en concentraciones entre 0,25 y $5 \mathrm{mg} \mathrm{L}^{-1}$. Se debe investigar cuál es el rango de concentraciones de EDTA necesario para que sea beneficioso en un cultivo y no constituya además, un gasto innecesario. Por otra parte, la utilización de las larvas de Petrolisthes laevigatus dado su sensibilidad, fácil recolecta y adaptabilidad al laboratorio, podrían ser investigados con el objeto de que sean incluidos en las Normas Chilenas como indicadores sensibles de contaminación por metales, como el cromo.

\section{Agradecimientos}

Este trabajo fue financiado parcialmente por el Comité Oceanográfico Nacional de Chile, a través del proyecto CONA-C12F 06-07.
Agradecemos al Sr. Patricio Alarcón M. por la edición de las fotografías, a los correctores y editor por su valioso aporte.

\section{Literatura citada}

Ahumada R \& J Vargas. 2005. Trace metals: inputs, sedimentation and accumulation in San Vicente Bay, Chile. Environmental Monitoring and Assessment 100: 11-22.

Albornoz L \& I Wehrtmann. 1996. Aspects of the reproductive biology of Petrolisthes laevigatus (Guerin, 1835) (Decapoda: Anomura: Porcellanidae). II. Description of the larval development, including the crab stage, under laboratory conditions. Archives of Fisheries and Marine Research 43: 137-157.

Altamirano-Chovar C, A Rudolph \& R Sepúlveda. 2006. Differential sensitivity to varying degrees of human influence in juvenile Semimytilus algosus (Gould, 1950) (Mollusca: Mytilidae), from four coastal sites in southcentral Chile. Bulletin of Environmental Contamination and Toxicology 77: 171-178.

Amín O. 1995. Toxicidad en invertebrados marinos de algunos metales pesados detectados en la zona costera próxima a Ushuaia, Tierra del Fuego. Ph D Thesis, University of Buenos Aires, Buenos Aires, 108 pp.

Antezana T, E Fagetti \& MT López. 1965. Observaciones bioecológicas en decápodos de Valparaíso. Revista de Biología Marina 12: 1-60.

Boada M, MA Moreno, H Gil, J Marcano \& J Maza. 2007. Heavy metals $\left(\mathrm{Cu}+{ }^{2}, \mathrm{Cd}^{2}, \mathrm{~Pb}^{2}, \mathrm{Zn}+{ }^{2}\right)$ in muscle and cephalothorax of wild shrimp (Litopenaeus schmitti, Farfantepenaeus subtilis, F. notialis and F. brasiliensis) from the Eastern region of Venezuela. Revista Científica, (Maracaibo) 17(2): 186-192.

Bodar C, C van Leeuwen, P Voogt \& D Zandee. 1990. Effect of cadmium on the reproduction strategy of Daphnia magna. Aquatic Toxicology 12: 301-310.

Caddy JF. 1989. Marine invertebrate fisheries. Their assessment and management, 752 pp. John Wiley \& Sons, New York.

Dorigan JV \& FL Harrison. 1987. Physiological responses of marine organisms to environmental stress, $501 \mathrm{pp}$. U.S. Department of Energy, Washington.

Espinoza C, R Diaz \& M Zuñiga. 2003. Toxicidad aguda y crónica de cobre y cadmio sobre dos especies de mitílidos: Perumytilus purpuratus y Aulacomya ater. Ciencia y Tecnología del Mar 26: 73-78.

Fingerman SW. 1985. Non-metal environmental pollutants and growth. En: Wenner AM (ed). Factors in adult growth, pp. 219-234. AA Balkema, Rotterdam.

Gagneten AM, S Gervasio \& JC Paggi. 2007. Heavy metal pollution and eutrophycation in the lower Salado River Basin (Argentina). Water Air Soil Pollution 178: 335-349. 
González-Pizani X, C Pastor \& E Dupré. 2006. Descripción del primer estadio larvario de Pachycheles chubutensis (Boschi, 1963) (Crustacea, Decapoda, Anomura) en Patagonia. Investigaciones Marinas 34(2): 191-196.

Hadfield MG \& MF Strathmann. 1996. Variability, flexibility and plasticity in life histories of marine invertebrates. Oceanologica Acta 19: 323-334.

Hernández P \& S Palma. 2003. Fecundidad, volumen del huevo y rendimiento reproductivo de cinco especies de porcelánidos intermareales del Norte de Chile (Decapoda, Porcellanidae). Investigaciones Marinas 31(2): 35-46.

Keselman D. 2002. Efectos del plomo sobre la embriogénesis y la eclosión larval de Chasmagnatus granulata (Decapoda, Brachyura). Tesis de Licenciatura en Ciencias Biológicas, Universidad de Concepción, Concepción, 89 pp.

Lardies M \& I Wehrtmann. 1996. Aspects of the reproductive biology of Petrolisthes laevigatus (Guérin, 1835) (Decapoda, Anomura, Porcellanidae). Part I: Reproductive output and chemical composition of eggs during embryonic development. Archives of Fishery and Marine Research 43: 121-135.

Lawrence AK, J Fox \& FL Castille. 1981. Decreased toxicity of coopper and manganese ions to shrimp nauplii (Penaeus stylirostris Stimpson) in the presence of EDTA. Journal of World Mariculture Society 12(1): 271-280.

Lee J, JH Park, YS Shin, BC Lee, NIK Chang, J Cho, SD Kim. 2009. Effect of dissolved organic matter on the growth of algae Pseudokirnereilla subcapitata, in Korean lakes: The importance of complexation reactions. Ecotoxicology and Environmental Safety 72: 375-343.

Levin LA. 2006. Recent progress in understanding larval dispersal: New directions and digressions. Integrative and Comparative Biology 46: 282-297

Lombardo RJ, L Ferrari \& JH Vinuesa. 1982. Efectos del etil parathion y DDVP sobre larvas de centolla Lithodes antarcticus Jacquinot. Ecosur 9(18): 141-151.

Moscoso J, A Rudolph, RD Sepúlveda \& C Suárez. 2006. Effect of temporary closure of the mouth of an estuary on the benthic macroinfauna: Lenga-Chile, a case study. Bulletin of Environmental Contamination and Toxicology 77: 484-491.

Ngah WSW, A Kamari \& S Fatinathan. 2006. Adsorption of chromium from aqueous solution using chitosan beads. Adsorption 12: 249-257.

Natale G, N Basso \& A Ronco. 2000. Effect of Cr (VI) on early life stages of three species of hylid frogs (Amphibia, Anura) from South America. Environmental Toxicology 15: 509-512.

Petriella AM \& EE Boschi. 1997. Crecimiento en crustáceos decápodos: resultados de investigaciones realizadas en Argentina. Investigaciones Marinas 25: 135-157.
Pinheiro MA \& A Fransozo. 1995. Fecundidade de Pachycheles haigae Rodríguez Da Costa, 1960 (Crustacea, Anomura, Porcellanidae) em Ubatuba (SP), Brasil. Revista Brasileira de Biología 55(4): 623-631.

Rai V \& S Mehrotra. 2008. Chromium-induced changes in ultramorphology and secondary metabolites of Phyllanthus amarus Schum and Thonn - hepatoprotective plant. Environmental Monitoring and Assessment 147: 307-315.

Rengaraj S, KH Yeon \& SH Moon. 2001. Removal of chromium from water and wastewater by ion exchange resin. Journal of Hazardous Materials B 87: 273-287.

Scelzo AM. 1997. Toxicidad del cobre en larvas nauplii del camarón comercial Artemesia longinaris Bate (Crustacea, Decapoda, Penaeidae). Investigaciones Marinas 25: 177185.

Scelzo AM. 1998. Efecto del EDTA(Ácido etilendiaminotetracético) sobre los desoves y larvas del camarón Artemesia longinaris (Crustacea, Decapoda, Penaeidae). Frente Marítimo 17C: 115120.

Schneider UA \& C Jefferson. 1997. Canadian water quality Guidelines for Chromium. Environmental Toxicology and Water Quality 12: 185-193.

Skoog DA, DM West, FJ Holler \& SR Crouch. 2005. Fundamentos de química analítica, 1175 pp. Thomson, México.

Sperling M \& B Welz. 1992. Determination of Cr (III) y Cr (VI) in water using flow injection on-line preconcentration with selective adsorption on activated alumina and flame atomic absorption spectrometric detection. Analytical Chemistry 64: 3101-3108.

Sridevi B \& SLN Reddy. 2000. Effect of trivalent and hexavalent chromium on carbohydrate metabolism of a freshwater field crab, Barytelphusa guerini. Environmental Monitoring and Assessment 61: 291-300.

StatSoft. Inc. 2001. Statistica. Data analysis software system [en línea]. <http://www.statsoft.com>

Threeprom J, S Purachaka \& L Potipan. 2005. Simultaneus determination of $\mathrm{Cr}$ (III)-EDTA and $\mathrm{Cr}$ (VI) by ion interaction chromatography using a C18 column. Journal of Chromatography A(1-2): 291-295.

Urrutia C, A Rudolph, MPLermanda \& R Ahumada. 2008. Assessment of EDTA in chromium (III-VI) toxicity on marine intertidal crab Petrolisthes laevigatus. Bulletin of Environmental Contamination and Toxicology 80: 526-528.

Vonesh J \& B Miner. 2004. Effects of fine grain environmental variability on morphological plasticity. Ecology Letters 7: 794-801.

Warnick S \& H Bell. 1969. The acute toxicity of some heavy metals to different species of aquatic insects. Journal of the Water Pollution Control Federation 41: 280-284. 